\title{
Quantum gravity with THESEUS
}

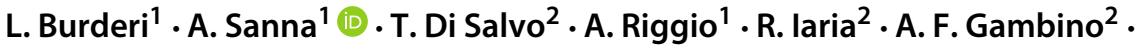 \\ A. Manca ${ }^{1}$ - A. Anitra ${ }^{2}$. S. M. Mazzola ${ }^{1}$ - A. Marino ${ }^{1}$
}

Received: 24 May 2021 / Accepted: 16 December 2021 / Published online: 20 January 2022

(C) The Author(s) 2022

\begin{abstract}
In this paper we explore the possibility to search for a dispersion law for light propagation in vacuo with a sample of Gamma-Ray Bursts detected by the THESEUS satellite. Within Quantum Gravity theories, different models for space-time quantization predict relative discrepancies of the speed of photons w.r.t. the speed of light that (in a series expansion) depend on a given power of the ratio of the photon energy to the Planck energy. This ratio is as small as $10^{-23}$ for photons in the soft $\gamma-$ ray band $(100 \mathrm{keV})$. The dominant effect is determined by the first significant term of this expansion. If the first order in this expansion is relevant, these theories imply a Lorentz Invariance Violation (LIV hereafter) and are generally dubbed LIV-theories. Therefore, to detect this effect, light must propagate over enormous distances and the experiment must have extraordinary sensitivity. Gamma-Ray Bursts, occurring at cosmological distances, could be used to detect this tiny signature of space-time granularity. Once the photons of a Gamma-Ray Burst are emitted at a given (cosmological) distance, they arrive on the detector with relative delays that linearly depends on the energy differences and on the distance travelled, that, given a set of cosmological parameters, is a unique function of the redshift. The strong temporal variability of the Gamma-Ray Bursts light-curves allows, with different techniques (e.g. crosscorrelations), to compute these delays by comparing light-curves of Gamma-Ray Bursts for which the redshift is known, in adjacent energy bands covering a sufficiently wide energy range. In this way, LIV-theories can be effectively constrained. THESEUS offers the opportunity to collect a homogeneous set of GRBs for which the redshift is known, with a signal to background ratio sufficient to compute delays through cross correlation techniques, and covering an energy band (from few keV to few $\mathrm{MeV}$ ) wide enough to produce significant delays. In this article we explore the possibility to constrain LIV-theories with THESEUS by means of Monte Carlo simulations. In summary, within the nominal duration of 3 years, THESEUS could constrain (or detect) Quantum Gravity Lorentz Invariance Violation effects at al level of 17 times the Planck Length $\left(1.6 \times 10^{-33} \mathrm{~cm}\right)$; if the mission is extended up to 7 years, this constrain is improved down to a level of 11 times the Planck Length.
\end{abstract}

\footnotetext{
L. Burderi

burderi@dsf.unica.it
} 
Keywords Gamma-ray bursts · X-rays · CubeSats · Nano-satellites ·

Temporal triangulation · Quantum gravity · Gravitational wave counterparts ·

All-sky monitor · Temporal triangulation

\section{GRB simulations and cross-correlation analysis}

We have used cross-correlation techniques to investigate the temporal delays between the light-curves of a given GRB in different energy bands. The light-curve of a bright long GRB observed by Fermi-GBM is shown in Fig. 1, Panel a. The bright Long GRB lasted for $\Delta t_{\mathrm{GRB}}=40 \mathrm{~s}$, with an average flux in the 50-300 keV energy band of $\phi_{\mathrm{GRB}}=6.5$ photons $/ \mathrm{s} / \mathrm{cm}^{2}$, and a background flux of $\phi_{\mathrm{BCK}}=2.8$ photons $/ \mathrm{s} / \mathrm{cm}^{2}$. Moreover, the GRB was characterised by variability on timescale of the order of $\sim 5$ ms.

Starting from this, we derived a template with millisecond resolution (see [1] for more details). Figure 1, Panel b, shows the detail of the main peak of the GRB template where the timescale of the fast variability is about $5 \mathrm{~ms}$. Using Monte-Carlo simulations, we generated light-curves as seen by detectors of different effective areas. Since the number of photons collected in a given energy energy band is a fraction of the total number of photons collected, light-curves obtained from detectors of different areas are equivalent - w.r.t. cross-correlation accuracies - to light-curves in different energy bands providing that the number of photons expected in a given energy band is equal to the number of photons detected with a detector of a given area. We performed cross-correlation analysis between pairs of simulated GRB with the aim to investigate the capability to reconstruct time delays between the observed signals. As an example, the cross-correlation function at $1 \mu$ s resolution for a pair of detectors of $100 \mathrm{~m}^{2}$ area is shown in Fig. 2, Panel a. Panel b shows the detail of the cross-correlation function around the peak and the best fit Gaussian. To determine a reliable estimation of the accuracy achievable using cross-correlation analysis, we
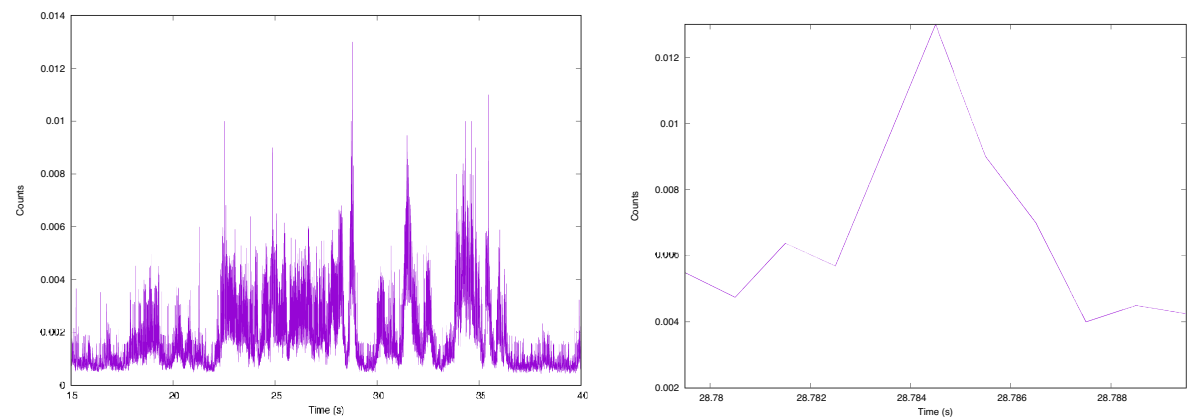

Fig. 1 GRB130502327 observed by Fermi-GBM. Panel a) GRB light-curve. Panel b) Template at one millisecond resolution (detail of the main peak) 
repeated the procedure described 1000 times, and we then fitted the distribution with a Gaussian model, from which we estimated an accuracy of $0.27 \mu$ s. Distributions for different effective areas, $56 \mathrm{~cm}^{2}$ (HERMES, see [2, 3] for more details on the HERMES project), $125 \mathrm{~cm}^{2}$ (Fermi-GBM), $1 \mathrm{~m}^{2}, 10 \mathrm{~m}^{2}, 50 \mathrm{~m}^{2}$, and $100 \mathrm{~m}^{2}$, are shown in the six panels of Fig. 3, top panel. The bottom panel shows the one sigma delay accuracy as a function of the effective area. The accuracy scales as the inverse of the effective area $A$ to the power of 0.6 , close but slightly better than the theoretical lower limit of 0.5 (derived from counting statistics).

The best fit formula is:

$$
\sigma_{\mathrm{cross}} \sim \sigma_{\Delta t}=3.3 \mu \mathrm{s} \times\left(\frac{A}{1 \mathrm{~m}^{2}}\right)^{-0.58} .
$$

In terms of the number of collected photons $N$ (adopting the same $0.8 / 6.5 \sim 40 \%$ overall background) we obtain:

$$
\sigma_{\text {cross }} \sim \sigma_{\Delta t}=3.3 \mu \mathrm{s} \times\left(\frac{N}{3.7 \times 10^{6}}\right)^{-0.58} .
$$

\section{A shallow dive into quantum gravity: minimal length hypothesis, Lorentz invariance violation, and dispersion relation for photons in vacuo}

Several theories proposed to describe quantum Space-Time, for instance some String Theories, predict the existence of a minimal length for space of the order of Planck length, $\ell_{\text {PLANCK }}=\sqrt{G \hbar / c^{3}}=1.6 \times 10^{-33} \mathrm{~cm}$ (see e.g. [4] for a review). This implies the following facts:

i) these theories predict a Lorentz Invariance Violation (LIV, hereafter). According to Special Relativity, a proper length, $\ell$, is Lorentz contracted by a factor
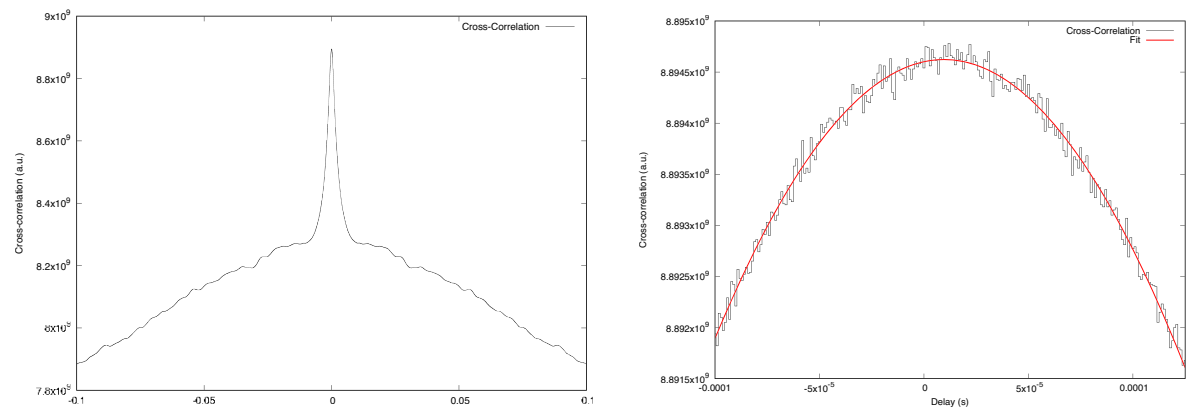

Fig. 2 Cross-correlation analysis of a simulated GRB seen by two identical detectors. Panel a) Crosscorrelation function. Panel $b$ ) Detail of the cross-correlation function at $1 \mu$ s around the main peak 

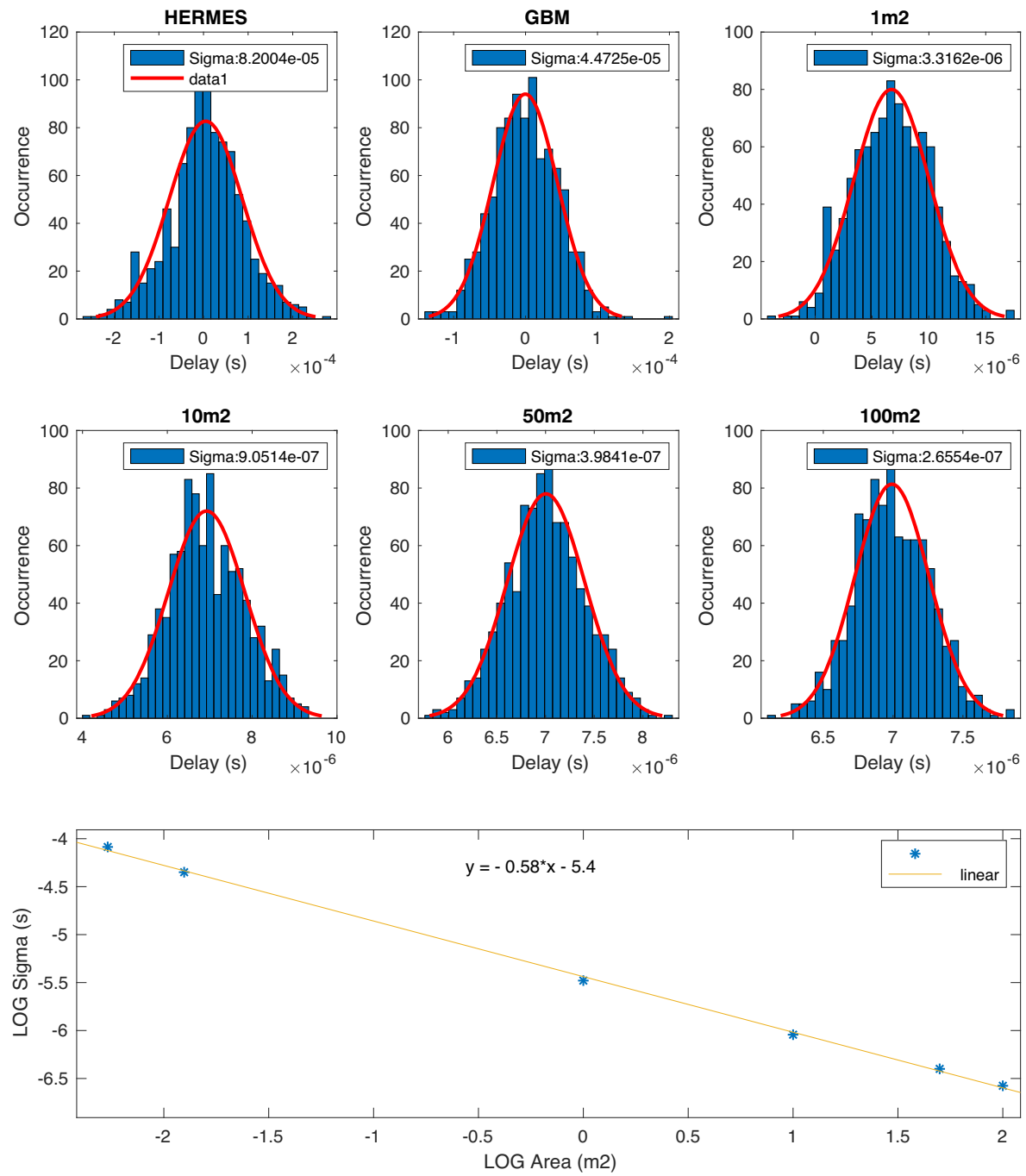

Fig. 3 Accuracy in cross correlation derived from Monte-Carlo simulations of GRBs. Top panel) Distributions of the delays obtained from cross-correlation analysis between 1000 pairs of simulated light-curves of identical detectors, for different effective areas, $56 \mathrm{~cm}^{2}$ (HERMES), $125 \mathrm{~cm}^{2}$ (Fermi-GBM), $1 \mathrm{~m}^{2}, 10$ $\mathrm{m}^{2}, 50 \mathrm{~m}^{2}$, and $100 \mathrm{~m}^{2}$. Bottom panel) Logarithmic plot of the one sigma delay accuracy as a function of the effective area and best linear fit

$\gamma^{-1}=\left[1-(v / c)^{2}\right]^{1 / 2}$ when observed from a reference system moving at speed $v$ w.r.t. the reference system in which $\ell$ is at rest. If $\ell_{\text {MIN }}=\alpha \ell_{\text {PLANCK }}$ (where $\alpha \sim 1$ is a dimensionless constant that depends on the particular theory under consideration) is the minimal length physically conceivable (in String Theories 
$\ell_{\text {MIN }}$ is the String length), no further Lorentz contraction must occur, at this scale. This is a violation of the Lorentz invariance.

ii) These theories predict the remarkable fact that the space has, somehow, the structure of a crystal lattice, at Planck scale.

iii) In perfect analogy with the propagation of light in crystals, these theories predict the existence of a dispersion law for photons in vacuo [5]. Since, for photons, energy scales as the inverse of the wavelength, this dispersion law can be expressed as a function of the energy of photons in units of the Quantum Gravity energy scale, which is the energy at which the quantum nature of gravity becomes relevant: $E_{\mathrm{QG}}=\zeta m_{\mathrm{PLANCK}} c^{2}=\zeta E_{\mathrm{PLANCK}}$, where $\zeta \sim \alpha^{-1} \sim 1$ is a dimensionless constant that depends on the particular theory under consideration, $m_{\text {PLANCK }}=\sqrt{c \hbar / G}=2.2 \times 10^{-5} \mathrm{~g}$ is the Planck mass, and the Planck energy is $E_{\text {PLANCK }}=1.2 \times 10^{19} \mathrm{GeV}$. We have:

$$
\left|v_{\mathrm{PHOT}} / c-1\right| \approx \xi\left(\frac{E_{\mathrm{PHOT}}}{\zeta m_{\mathrm{PLANCK}} c^{2}}\right)^{n}
$$

where $\xi \sim 1$ is a dimensionless constant that depends on the particular theory under consideration, $v_{\mathrm{PHOT}}$ is the group velocity of the photon wave-packet, and $E_{\mathrm{PHOT}}$ is the photon energy. The index $n$ is the order of the first relevant term in the expansion in the small parameter $\epsilon=E_{\mathrm{PHOT}} /\left(\zeta m_{\mathrm{PLANCK}} c^{2}\right)$. In several theories that predict the existence of a minimal length, typically, $n=1$. Finally, the modulus is present in (3) takes into account the possibility (predicted by different LIV theories) that higher energy photons are faster or slower than lower energy photons (discussed as sub-luminal, +1 , or super-luminal, -1 , as in [6]).

We stress that not all the theories proposed to quantise gravity predict a LIV at some scale. This is certainly the case for Loop Quantum Gravity (see e.g. [7-9]). No LIV is expected as a consequence of the recently proposed Space-Time Uncertainty Principle [10] and in the Quantum Space-Time [11]. In some of these theories it is possible to conceive a photon dispersion relation that does not violate Lorentz invariance, although the first relevant term is quadratic in the ratio photon energy over $E_{\mathrm{QG}}$, i.e. $n=2$ in this case. We explicitly note that, since $E_{\mathrm{QG}} \sim 10^{19}$ $\mathrm{GeV}$, second order effects are almost not relevant even for photons of at $0.1 \mathrm{PeV}$ energies $\left(10^{14} \mathrm{eV}\right)$, the highest energy photons ever recorded, recently confirmed to be emitted by the Crab Nebula [12]. Indeed also for these extreme photons $\left(E_{\mathrm{PHOT}} / E_{\mathrm{QG}}\right)^{2} \sim 10^{-28}$.

\subsection{Dispersion relation for photons in vacuo}

During motion at constant velocity, travel time is the ratio between the distance travelled $D_{\text {TRAV }}$ and the speed. Therefore, differences in speed result in differences in the arrival times $\Delta t_{\mathrm{QG}}$ of photons of different energies $\Delta E_{\mathrm{PHOT}}$ departing from the same point at the same time, such as those emitted during a GRB event. For small speed differences, as those predicted by the dispersion relations discussed above, these delays 
scales with the same order $n-$ in the ratio $\Delta E_{\mathrm{PHOT}} / E_{\mathrm{QG}}-$ as that between photon energy and Quantum Gravity energy scale:

$$
\Delta t_{\mathrm{QG}}= \pm \xi\left(\frac{D_{\mathrm{TRAV}}}{c}\right)\left(\frac{\Delta E_{\mathrm{PHOT}}}{\zeta m_{\mathrm{PLANCK}} c^{2}}\right)^{n}
$$

where $\xi \sim 1$ is a dimensionless constant that depends on the particular theory under consideration and the sign \pm takes into account the possibility (predicted by different LIV theories) that higher energy photons are faster or slower than lower energy photons respectively, as discussed above [6].

On the other hand, the distance traveled has to take into account the cosmological expansion, being a function of cosmological parameters and redshift. The comoving trajectory of a particle is obtained by writing its Hamiltonian in terms of the comoving momentum [13]. The computation of the delays has to take into account the fact that the proper distance varies as the universe expands. Photons of different energies are affected by different delays along the path, so, because of cosmological expansion, a delay produced further back in the path amounts to a larger delay on Earth. Taking into account these effects this modified "distance traveled" DEXP can be computed [13].

More specifically we adopted the so called Lambda Cold Dark Matter Cosmology $(\Lambda \mathrm{CDM})$ with the following values [14]: $H_{0}=67.74(46) \mathrm{km} \mathrm{s}^{-1} \mathrm{Mpc}^{-1}, \Omega_{\mathrm{k}}=0$, curvature $k=0$ that implies a flat Universe, $\Omega_{\mathrm{R}}=0$, radiation $=0$ that implies a cold Universe, $w=-1$, negative pressure Equation of State for the so called Dark Energy that implies an accelerating Universe, $\Omega_{\Lambda}=0.6911(62)$ and $\Omega_{\text {Matter }}=$ $0.3089(62)$ (see [14], for the parameters and related uncertainties).

With these values we have:

$$
\frac{D_{\mathrm{EXP}}}{c}=\frac{1}{H_{0}} \int_{0}^{z} d z \frac{(1+z)}{\sqrt{\Omega_{\Lambda}+(1+z)^{3} \Omega_{\mathrm{Matter}}}},
$$

where $z$ is the redshift.

Substituting $D_{\text {TRAV }}$ of (4) with $D_{\text {EXP }}$ derived in (5) we finally obtain the delays between the time of arrival of photons of different energies as a function of the specific Dispersion Relation adopted, the specific Cosmology adopted, and the redshift:

$$
\Delta t_{\mathrm{QG}}= \pm \xi\left(\frac{1}{H_{0}} \int_{0}^{z} d z \frac{(1+z)}{\sqrt{\Omega_{\Lambda}+(1+z)^{3} \Omega_{\mathrm{Matter}}}}\right)\left(\frac{\Delta E_{\mathrm{PHOT}}}{\zeta m_{\mathrm{PLANCK}} c^{2}}\right)^{n}
$$

\subsection{Computation of the expected delays: long GRB at different redshifts}

We considered a bright Long GRB lasted for $\Delta t_{\mathrm{GRB}}=40 \mathrm{~s}$, with average flux in the $50-300 \mathrm{keV}$ energy band $\phi_{\mathrm{GRB}}=6.5$ photons $/ \mathrm{s} / \mathrm{cm}^{2}$, background flux of $\phi_{\mathrm{BCK}}=$ 2.8 photons $/ \mathrm{s} / \mathrm{cm}^{2}$, and variability timescale $\sim 5 \mathrm{~ms}$ discussed in Section 1 . 
We selected eight consecutive energy bands from 5 to $50 \mathrm{MeV}$. For an overall collecting area of $100 \mathrm{~m}^{2}$, the number of detected photons in each band was computed adopting a Band function, an empirical function that well fits GRB spectra [16]:

$$
\frac{d N_{E}(E)}{d A d t}=F \times \begin{cases}\left(\frac{E}{E_{\mathrm{B}}}\right)^{\alpha} \exp \left\{-(\alpha-\beta) E / E_{\mathrm{B}}\right\}, & E \leq E_{\mathrm{B}} \\ \left(\frac{E}{E_{\mathrm{B}}}\right)^{\beta} \exp \{-(\alpha-\beta)\}, & E \geq E_{\mathrm{B}},\end{cases}
$$

where $E$ is the photon energy, $d N_{E}(E) /(d A d t)$ is the photon intensity energy distribution in units of photons $/ \mathrm{cm}^{2} / \mathrm{s} / \mathrm{keV}, F$ is a normalisation constant in units of photons $/ \mathrm{cm}^{2} / \mathrm{s} / \mathrm{keV}, E_{\mathrm{B}}$ is the break energy, and $E_{\mathrm{P}}=[(2+\alpha) /(\alpha-\beta)] E_{\mathrm{B}}$ is the peak energy. For most GRBs: $\alpha \sim-1, \beta \sim-2.0 \div-2.5$, and $E_{\mathrm{B}} \sim 225 \mathrm{keV}$ that implies $E_{\mathrm{P}} \sim 150 \mathrm{keV}$. We considered soft and hard cases $(\beta=-2.5$ and $\beta=-2.0$, respectively). Once the number of photons collected in each band $N$ is computed, the one sigma accuracy in the delays of the ToA of photons in a given energy band, $E_{C C}(N)$, is computed adopting the results of cross-correlation analysis performed on pairs of Monte-Carlo simulated GRBs in Section 1 and expressed in (2) adopting the most conservative assumption that $E_{C C}(N)$ scales as $\left(N / 3.7 \times 10^{6}\right)^{-0.5}$ (as expected from counting statistics) and not as $\left(N / 3.7 \times 10^{6}\right)^{-0.58}$ of (2). We adopted the geometric mean of the lower and upper limits of a given energy band, $E_{\min }$ and $E_{\max }$ respectively, as representative of the average energy of the photons in that given band $E_{\mathrm{AVE}}=\sqrt{E_{\min } \times E_{\max }}$. With this, the energy difference between photons of different energy bands w.r.t. photons of very low energy $E_{\mathrm{AVE}} \sim 0$, are $\Delta E_{\mathrm{PHOT}}=E_{\mathrm{AVE}}$. We adopted the cosmology described in Section 2.1, a first order dispersion relation i.e. $n=1$, and, finally, $\xi=1$ and $\zeta=1$. The Quantum Gravity delays of the time of arrival of photons of different energy bands were computed with (6), for values of the redshift $z=0.1,0.5,1.0,3.0$, typical of GRBs as shown in Fig. 4. The results are shown in Table 1 . Numbers in red and blue refer to delays below

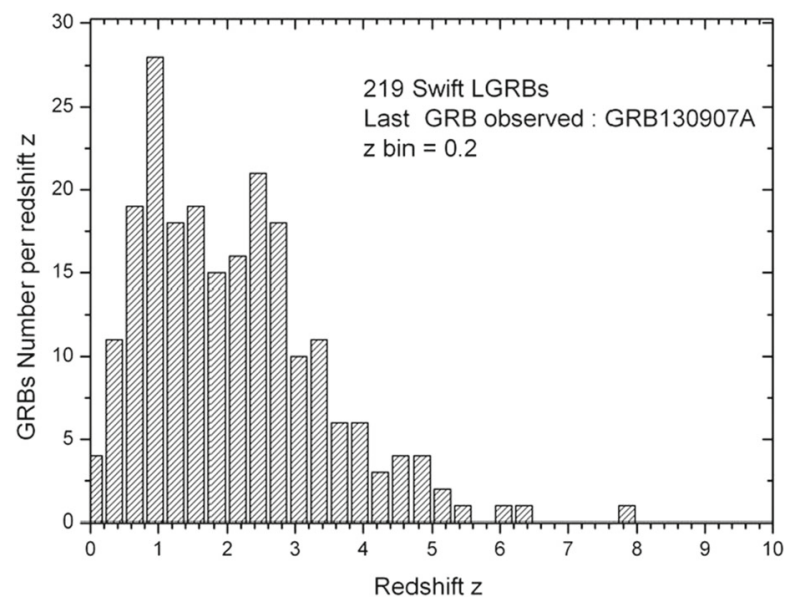

Fig. 4 Distribution of 219 GRBs detected by Swift as a function of the redshift in bins of $\Delta z=0.2$. Figure from [15] 
Table 1 Photon fluence and expected delays induced by a Quantum Gravity first order Dispersion Relation for the bright Long GRB described in Section 1 and observed with a detector of cumulative effective area of $100 \mathrm{~m}^{2}$ (e.g. obtained by adding the photons collected by $N=10^{4}$ nano-satellites of $100 \mathrm{~cm}^{2}$ each)

Quantum Gravity delays predicted with a first order photon dispersion relation

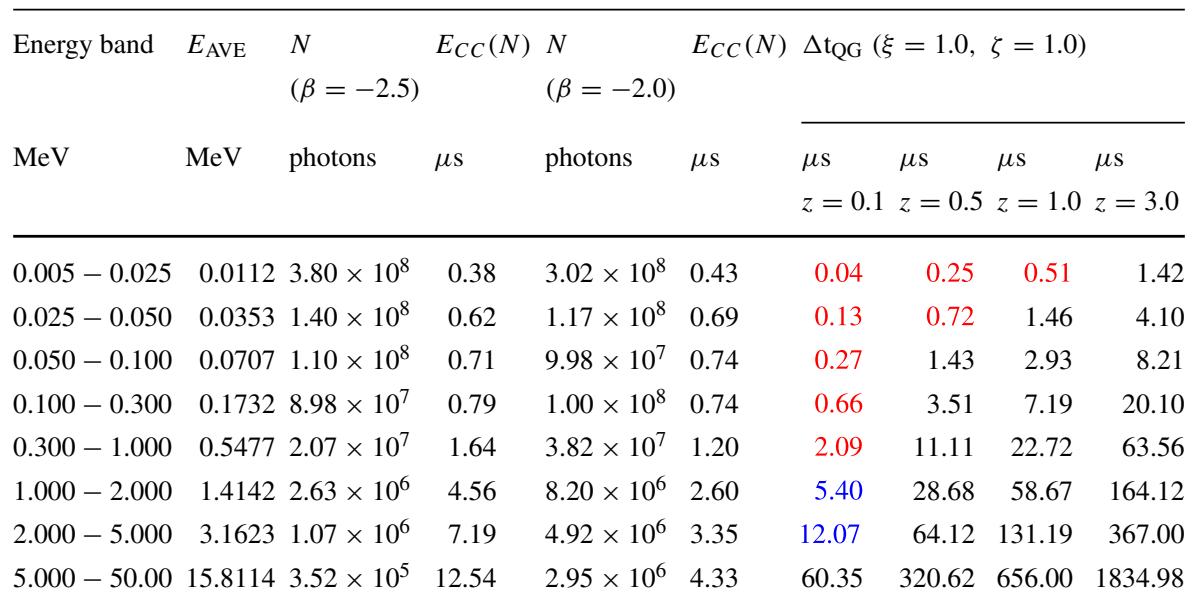

The GRB is described by a Band function with $\alpha=-1, \beta=-2.5 \div-2.0, E_{\mathrm{B}} \sim 225 \mathrm{keV}$. The modified "distance traveled" by the photons $D_{\text {EXP }}$ described in the text has been computed for each redshift adopting a $\Lambda$ CDM cosmology with $\Omega_{\Lambda}=0.6911$ and $\Omega_{\text {Matter }}=0.3089$. This implies the following: $D_{\operatorname{EXP}}=453.9$ $\mathrm{Mpc}$ for $z=0.1, D_{\mathrm{EXP}}=2411.4 \mathrm{Mpc}$ for $z=0.5, D_{\mathrm{EXP}}=4933.6 \mathrm{Mpc}$ for $z=1.0, D_{\mathrm{EXP}}=13801.2$ Mpc for $z=3.0$. Adopting $n=1, \xi=1$ and $\zeta=1$, we found $\left|\Delta t_{\mathrm{QG}}\right|=3.8168 \mu \mathrm{s} \times \Delta E_{\mathrm{PHOT}} /(1 \mathrm{MeV})$ for $z=0.1,\left|\Delta t_{\mathrm{QG}}\right|=20.2775 \mu \mathrm{s} \times \Delta E_{\mathrm{PHOT}} /(1 \mathrm{MeV})$ for $z=0.5,\left|\Delta t_{\mathrm{QG}}\right|=41.4863 \mu \mathrm{s} \times \Delta E_{\mathrm{PHOT}} /(1$ $\mathrm{MeV})$ for $z=1.0,\left|\Delta t_{\mathrm{QG}}\right|=116.0544 \mu \mathrm{s} \times \Delta E_{\mathrm{PHOT}} /(1 \mathrm{MeV})$ for $z=3.0 . \Delta E_{\mathrm{PHOT}}=E_{\mathrm{AVE}}=$ $\sqrt{\mathrm{E}_{\max } \times \mathrm{E}_{\min }}$ (see text). The (statistical) cross-correlation accuracies are computed as $E_{C C}(N)=3.3$ $\mu \mathrm{s} \sqrt{3.710^{6} / N}$, obtained from Monte-Carlo simulations

and just above one sigma accuracy, respectively. Numbers in black are above three sigma.

\subsection{Intrinsic delays or quantum gravity delays?}

Because of unknown details on the Fireball model, intrinsic delays in the emission of photons of different energy bands are possible. For a given GRB, these intrinsic delays can mix to, or even mimic, a genuine quantum gravity effect, making its detection impossible. However, intrinsic delays in the emission mechanism are independent of the distance of the GRB. On the other hand, the delays induced by a photon dispersion law are proportional both to the distance traveled (known function of redshift) and to the differences in energy of the photons. This double dependence on energy and redshift is the unique signature of a genuine Quantum Gravity effect. This behaviour, shown in Table 1, demonstrates that, given an adequate collecting area, GRBs are indeed excellent tools to effectively search for a first order dispersion law for photons, once their redshifts are known. 


\section{Testing LIV theories with THESEUS}

\subsection{The sample}

We considered a sample of long GRBs detectable by THESEUS in one year of mission as derived from the mock catalogue of 2 millions of long GRB produced by Ghirlanda et al. (see Ghirlanda et al., 2021, for details). Moreover we considered the fraction of long GRBs in the adopted mock catalogue that are detectable by THESEUS for which a measure of the redshift is obtainable according to the sensitivity of the mission and the conditions under which a good redshift measurement is possible (see Mereghetti et al., 2021, for details). This gave us a sample of about 200 long GRBs per year. In order to apply the accuracy in delays of (2), derived in the hypothesis of a signal-to-background ratio $\sim 2$, we further selected the GRBs that fulfil these criteria ( $\sim 4$ per year). It is possible, in principle, to use the whole sample of 200 GRBs per year, however, for GRBs with signal-to-background ratio $<2$, the accuracy in computing the delays has a much more complex dependence on the number of photons in the GRB, than that expressed in (2). In this case further studies are required to obtain a reliable relation that express the accuracy in computing the delays as a function of the number of photons in the GRB and the number of photons in the background. The inclusion of the whole sample of GRBs in our analysis is possible once this more complex relation is adequately investigated, but this is beyond the scope of this short report and will be discussed elsewhere. Finally we considered two scenarios: a mission lasting for its nominal duration, 3 years lifetime (13 GRBs in the sample), and an extended mission, lasting for 7.5 years (27 GRBs in the sample). ${ }^{1}$

\subsection{The analysis}

We firstly explored, for each GRB in a given energy band, $\Delta E_{\mathrm{PHOT}} \mathrm{i}, i=$ $1, \ldots, N_{\text {bands }}$, where $N_{\text {bands }}$ is the total number of energy bands adopted, the dependence of the delays on the redshift. This, according to (6) with $n=1$ and $\xi \sim 1$ is:

$$
\Delta t_{\mathrm{QG}} \mathrm{i}=\frac{1}{H_{0}}\left(\frac{\Delta E_{\mathrm{PHOT}} \mathrm{i}}{\zeta m_{\mathrm{PLANCK}} c^{2}}\right) \times f(z)
$$

where

$$
f(z)=\int_{0}^{z} d z \frac{(1+z)}{\sqrt{\Omega_{\Lambda}+(1+z)^{3} \Omega_{\text {Matter }}}} .
$$

Fits of (8) as a function of $f(z)$, are shown in Fig. 5, for the eight energy bands chosen to cover the whole energy range of THESEUS, from $10 \mathrm{keV}$ to $10 \mathrm{MeV}$. In particular these energy bands are: 2-25 keV; 25-50 keV; 50-150 keV; 150-300 keV; 300-1000 keV; 1000-2000 keV; 2000-5000 keV; 5000-10000 keV.

\footnotetext{
${ }^{1}$ The datasets generated during and/or analysed during the current study are available from the corresponding author on reasonable request.
} 

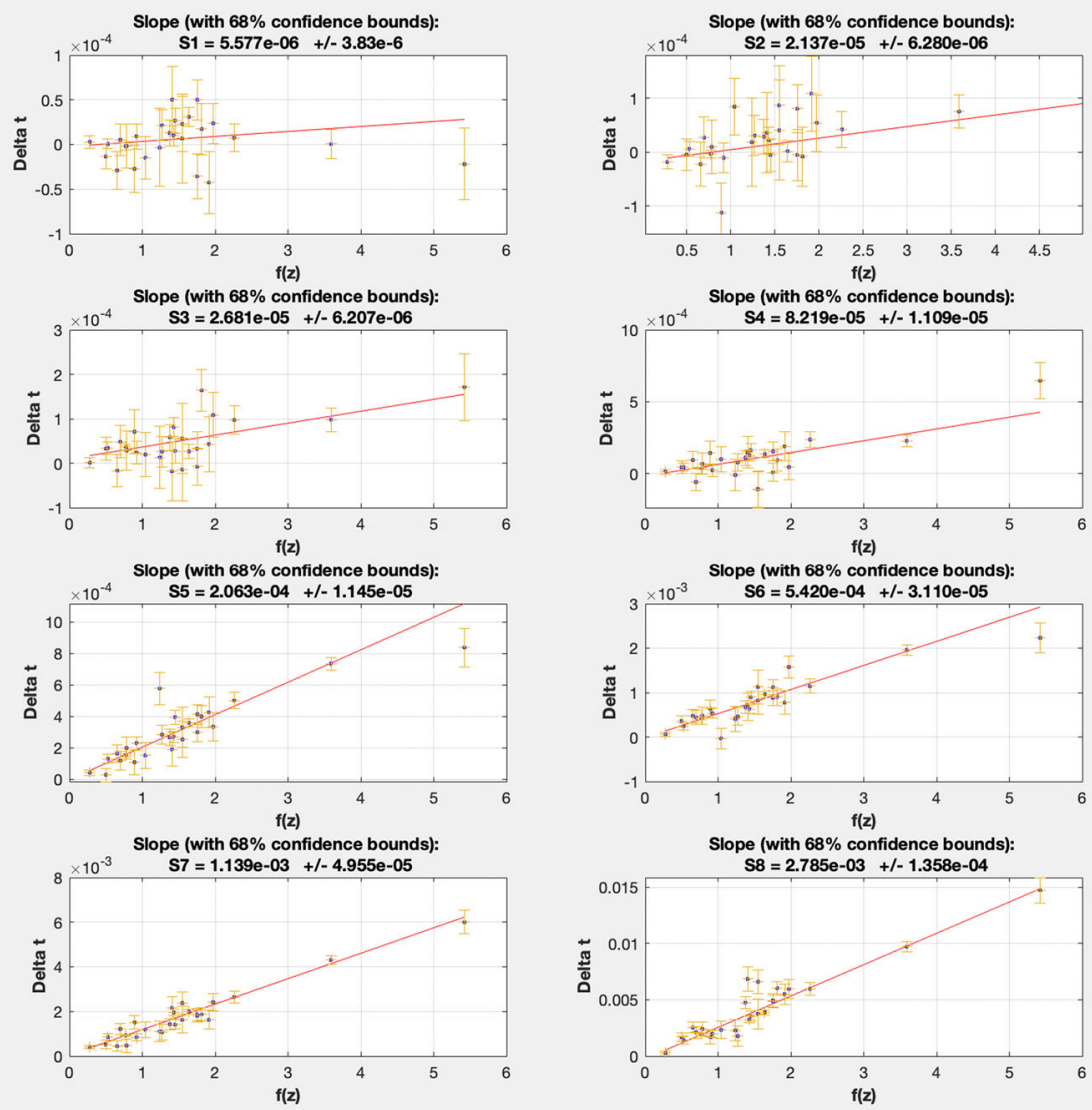

Fig. 5 The eight panels show the delays $H_{0}^{-1}\left(\Delta E_{\text {PHOT i }} /\left(\zeta m_{\text {PLANCK }} c^{2}\right)\right)$ (i.e. the expected delays normalised to a redshift function $f(z)=1$ ) vs. the redshift function $f(z)$ defined in (9) in the text, for the eight energy bands chosen to cover the whole energy range of THESEUS, from $10 \mathrm{keV}$ to $10 \mathrm{MeV}$. The best fit slope of each panel are the values $s_{\mathrm{i}}$ defined in (10) in the text

The slopes of these linear fits represent the eight values of the quantities

$$
s_{\mathrm{i}}=\frac{1}{\zeta} \frac{1}{H_{0}} g\left(E_{\text {PHOT i }}\right)
$$

where

$$
g\left(E_{\mathrm{PHOT}} \mathrm{i}\right)=\left(\frac{\Delta E_{\mathrm{PHOT}} \mathrm{i}}{m_{\mathrm{PLANCK}} c^{2}}\right)
$$

for $i=1, \ldots, N_{\text {bands. }}$. Fit of (10) as a function of $h\left(E_{\mathrm{PHOT}}\right.$ i $)=g\left(E_{\mathrm{PHOT}}\right.$ i $) / H_{0}$, are shown in Fig. 5, for the eight energy bands chosen to cover the whole energy range of THESEUS, from $10 \mathrm{keV}$ to $10 \mathrm{MeV}$. The slope of this linear fit gives the quantity

$$
\mu=\frac{1}{\zeta}
$$


As it is customary in this field, the parameter $\mu$ express the strength of the LIV effect. The higher the $\mu$, the greater the effect of Quantum Gravity, in the sense that a value of e.g. $\mu=5$ implies that the effects of Quantum Gravity are already relevant at energies equal to $1 / 5$ of Planck energy, $m_{\mathrm{PLANCK}} c^{2}$.

To quantify the capability of THESEUS in deriving a robust upper limit (or detection) of a LIV effect, we injected, in our sample, a LIV parametrised by a dummy value of $\mu$. In particular we adopted $\mu=30$ in the simulations performed. The three sigma upper limit on $\mu$ has been computed from the one sigma error on the slope quoted in the fit.

To be conservative, in performing this last fit we made two assumptions:

i) we artificially amplified by one order of magnitude the statistical errors on the quantities $s_{\mathrm{i}}$ w.r.t. obtained by applying (2) derived from our Monte-Carlo simulations of long GRBs. This is done to take into account several effects that could worsen the statistical accuracies. Among these the most relevant is the unknown (a priori) variation of the shape of the GRB light-curve in different energy bands that could bias the value of the cross correlation;
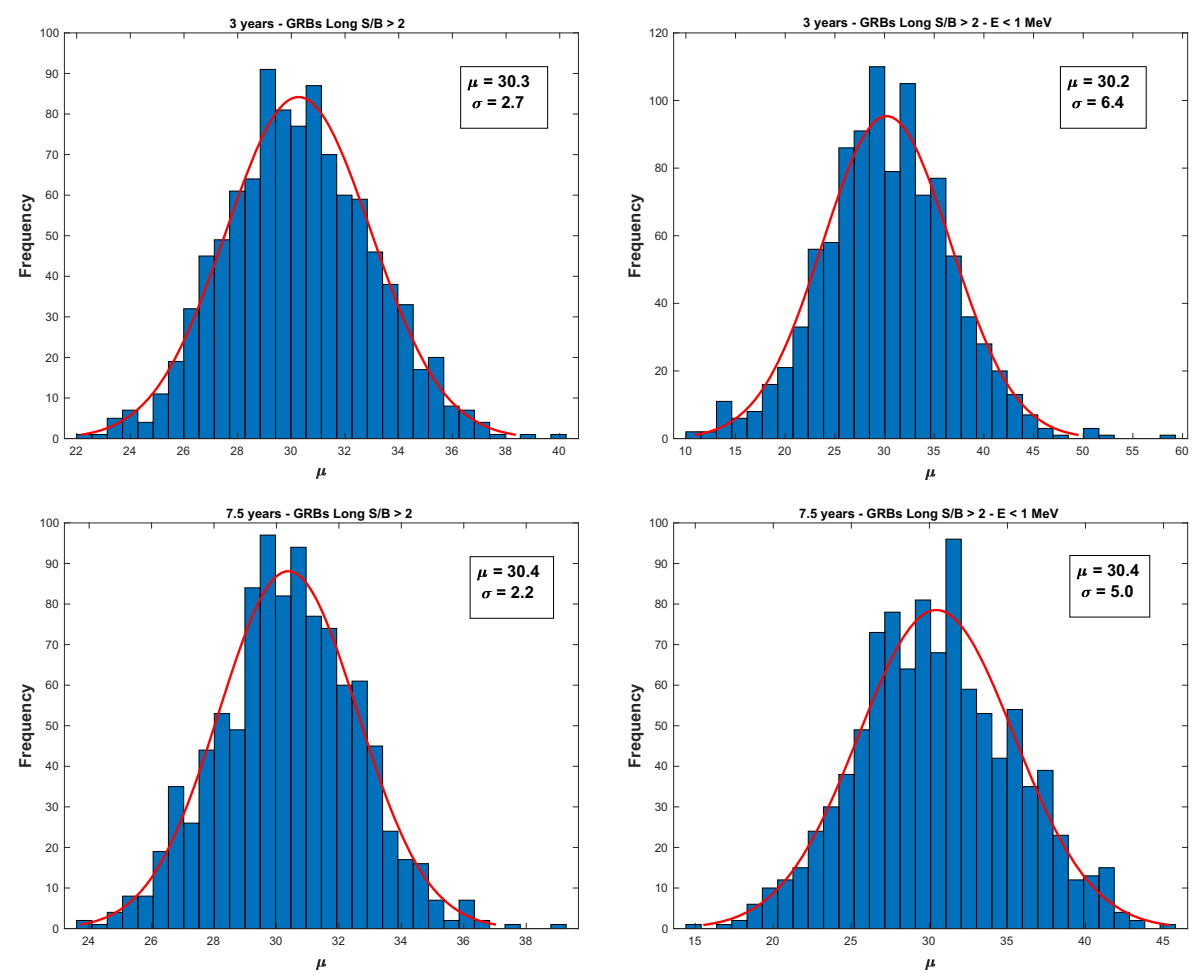

Fig. 6 Distributions of the Monte-Carlo simulations performed to explored the $\mu$ parameter for the two mission scenarios described in the text: i.e. nominal mission duration, 3 years (13 GRBs in the sample), and an extended mission, 7.5 years (27 GRBs in the sample). We adopted $\mu=30$ for generating a dummy LIV effect and, as an upper limit to the value of the $\mu$ parameter, three times the one sigma uncertainty obtained from the distribution of 1000 Monte-Carlo simulations 
ii) we considered two scenarios, the first in which the similarity of the light-curves is guaranteed over the whole THESEUS energy band $2 \mathrm{keV}-10 \mathrm{MeV}$. In this case all the $s_{\mathrm{i}}$ were included in our analysis. In a more conservative approach, we assumed that the similarity of the light-curves holds only for energies $\leq 1$ $\mathrm{MeV}$ and, consequently, all the $s_{\mathrm{i}}$ above this threshold were excluded from our analysis.

Our results are shown in Fig. 6 where we explored the two scenarios mentioned above, namely a mission lasting for its nominal duration, 3 years (13 GRBs in the sample), and an extended mission, lasting for 7.5 years (27 GRBs in the sample).

We compare our results with and [17] who set a robust constraint on LIV using Fermi-LAT GRB data. Applying different estimations procedures developed on the basis of statistical measures to the eight observed GRBs relatively bright in multi$\mathrm{GeV}$ energies detected by Fermi-LAT, they constrained $\mu \geq 50$ and $\mu \geq 15$ depending on different hypotheses and statistical methods adopted. For a mission lasting for its nominal duration, 3 years, the results are a three sigma upper limit on $\mu \leq 9$, considering the whole energy range (2 keV-10 MeV) and a three sigma upper limit on $\mu \leq 20$, considering an energy range $2 \mathrm{keV}-1 \mathrm{MeV}$. For a mission lasting for an extended duration, 7.5 years, the results are a three sigma upper limit on $\mu \leq 7$, considering the whole energy range (2 keV-10 $\mathrm{MeV}$ ) and a three sigma upper limit on $\mu \leq 15$, considering an energy range $2 \mathrm{keV}-1 \mathrm{MeV}$.

Acknowledgements This work has been carried out in the framework of the HERMES-TP and HERMES-SP collaborations. We acknowledge support from the European Union Horizon 2020 Research and Innovation Framework Program under grant agreement HERMES-Scientific Pathfinder n. 821896 and from ASI-INAF Accordo Attuativo HERMES Technologic Pathfinder n. 2018-10-H.1-2020. LB and AS acknowledge financial contribution from the PRIN 2017 agreement n. 20179ZF5KS. LB, AS and AR acknowledge financial contribution from the FdS 2017, CUP n. F71117000150002.

Open Access This article is licensed under a Creative Commons Attribution 4.0 International License, which permits use, sharing, adaptation, distribution and reproduction in any medium or format, as long as you give appropriate credit to the original author(s) and the source, provide a link to the Creative Commons licence, and indicate if changes were made. The images or other third party material in this article are included in the article's Creative Commons licence, unless indicated otherwise in a credit line to the material. If material is not included in the article's Creative Commons licence and your intended use is not permitted by statutory regulation or exceeds the permitted use, you will need to obtain permission directly from the copyright holder. To view a copy of this licence, visit http://creativecommons.org/licenses/by/4.0/.

\section{References}

1. Sanna, A., et al.: Timing techniques applied to distributed modular high-energy astronomy: the hermes project. Society of Photo-Optical Instrumentation Engineers (SPIE) Conference Series (2020)

2. Fiore, F., Burderi, L., Lavagna, M., Bertacin, R., Evangelista, Y., Campana, R., Fuschino, F., Lunghi, P., Monge, A., Negri, B., Pirrotta, S., Puccetti, S., Sanna, A., Amarilli, F., Ambrosino, F., AmelinoCamelia, G., Anitra, A., Auricchio, N., Barbera, M., Bechini, M., Bellutti, P., Bertuccio, G., Cao, J., Ceraudo, F., Chen, T., Cinelli, M., Citossi, M., Clerici, A., Colagrossi, A., Curzel, S., Della Casa, 
G., Demenev, E., Del Santo, M., Dilillo, G., Di Salvo, T., Efremov, P., Feroci, M., Feruglio, C., Ferrandi, F., Fiorini, M., Fiorito, M., Frontera, F., Gacnik, D., Galgoczi, G., Gao, N., Gambino, A.F., Gandola, M., Ghirlanda, G., Gomboc, A., Grassi, M., Guzman, A., Karlica, M., Kostic, U., Labanti, C., La Rosa, G., Lo Cicero, U., Lopez-Fernandez, B., Malcovati, P., Maselli, A., Manca, A., Mele, F., Milankovich, D., Morgante, G., Nava, L., Nogara, P., Ohno, M., Ottolina, D., Pasquale, A., Pal, A., Perri, M., Piazzolla, R., Piccinin, M., Pliego-Caballero, S., Prinetto, J., Pucacco, G., Rashevsky, A., Rashevskaya, I., Riggio, A., Ripa, J., Russo, F., Papitto, A., Piranomonte, S., Santangelo, A., Scala, F., Sciarrone, G., Selcan, D., Silvestrini, S., Sottile, G., Rotovnik, T., Tenzer, C., Troisi, I., Vacchi, A., Virgilli, E., Werner, N., Wang, L., Xu, Y., Zampa, G., Zampa, N., Zanotti, G.: The HERMEStechnologic and scientific pathfinder. In: Society of Photo-Optical Instrumentation Engineers (SPIE) Conference Series, Society of Photo-Optical Instrumentation Engineers (SPIE) Conference Series, vol. 11444, pp 114441R (2020)

3. Burderi, L., Di Salvo, T., Riggio, A., Gambino, A.F., Sanna, A., Fiore, F., Amarilli, F., Amati, L., Ambrosino, F., Amelino-Camelia, G., Anitra, A., Barbera, M., Bechini, M., Bellutti, P., Bertaccin, R., Bertuccio, G., Campana, R., Cao, J., Capozziello, S., Ceraudo, F., Chen, T., Cinelli, M., Citossi, M., Clerici, A., Colagrossi, A., Costa, E., Curzel, S., De Laurentis, M., Della Casa, G., Della Valle, M., Demenev, E., Del Santo, M., Dilillo, G., Efremov, P., Evangelista, Y., Feroci, M., Ferruglio, C., Ferrandi, F., Fiorini, M., Fiorito, M., Frontera, F., Fuschino, F., Gacnik, D., Galgoczi, G., Gao, N., Gandola, M., Ghirlanda, G., Gomboc, A., Grassi, M., Guidorzi, C., Guzman, A., Iaria, R., Karlica, M., Kostic, U., Labanti, C., La Rosa, G., Lo Cicero, U., Lopez Fernandez, B., Lunghi, P., Malcovati, P., Maselli, A., Manca, A., Mele, F., Milankovich, D., Monge, A., Morgante, G., Nava, L., Negri, B., Nogara, P., Ohno, M., Ottolina, D., Pasquale, A., Pal, A., Perri, M., Piccinin, M., Piazzolla, R., Pirrotta, S., Pliego-Caballero, S., Prinetto, J., Pucacco, G., Puccetti, S., Rapisarda, M., Rashevskaya, I., Rashevsky, A., Ripa, J., Russo, F., Papitto, A., Piranomonte, S., Santangelo, A., Scala, F., Sciarrone, G., Selcan, D., Silvestrini, S., Sottile, G., Rotovnik, T., Tenzer, C., Troisi, I., Vacchi, A., Virgilli, E., Werner, N., Wang, L., Xu, Y., Zampa, G., Zampa, N., Zane, S., Zanotti, G.: Grailquest and HERMES: hunting for gravitational wave electromagnetic counterparts and probing space-time quantum foam. In: Society of Photo-Optical Instrumentation Engineers (SPIE) Conference Series, Society of PhotoOptical Instrumentation Engineers (SPIE) Conference Series, vol. 11444, pp. 114444Y (2020)

4. Hossenfelder, S.: Minimal length scale scenarios for quantum gravity. Living Rev. Relativ. 16, 2 (2013)

5. Amelino-Camelia, G.: Are We at the Dawn of Quantum-Gravity Phenomenology? vol. 541 of Lecture Notes in Physics, 1 (2000)

6. Amelino-Camelia, G., Smolin, L.: Prospects for constraining quantum gravity dispersion with near term observations. Phys. Rev. D 80, 084017 (2009)

7. Rovelli, C., Smolin, L.: Knot theory and quantum gravity. Phys. Rev. Lett. 61, 1155-1158 (1988)

8. Rovelli, C., Smolin, L.: Loop space representation of quantum general relativity. Nucl. Phys. B 331, 80-152 (1990)

9. Rovelli, C.: Loop quantum gravity. Living Rev. Relativ. 1, 1 (1998)

10. Burderi, L., Di Salvo, T., Iaria, R.: Quantum clock: a critical discussion on spacetime. Phys. Rev. D 93, 064017 (2016)

11. Sanchez, N.G.: New quantum structure of space-time. Gravit. Cosmol. 25, 91-102 (2019)

12. Amenomori, M., Bao, Y.W., Bi, X.J., Chen, D., Chen, T.L., Chen, W.Y., Chen, X., et al.: First detection of photons with energy beyond $100 \mathrm{TeV}$ from an astrophysical source. Phys. Rev. Lett. 123, 051101 (2019)

13. Jacob, U., Piran, T.: Lorentz-violation-induced arrival delays of cosmological particles. J. Cosmol. Astropart. Phys. 2008, 031 (2008)

14. Planck Collaboration, et al.: Planck 2015 results. XIII. Cosmological parameters. A\&A 594, A13 (2016)

15. Zitouni, H., Guessoum, N., Azzam, W.J.: Revisiting the Amati and Yonetoku correlations with Swift GRBs. Ap\&SS 351, 267-279 (2014)

16. Band, D., Matteson, J., Ford, L., Schaefer, B., Palmer, D., Teegarden, B., Cline, T., Briggs, M., Paciesas, W., Pendleton, G., Fishman, G., Kouveliotou, C., Meegan, C., Wilson, R., Lestrade, P.: BATSE observations of gamma-ray burst spectra. I. Spectral diversity. Astrophysical Journal 413, 281 (1993) 
17. Ellis, J., Konoplich, R., Mavromatos, N.E., Nguyen, L., Sakharov, A.S., Sarkisyan-Grinbaum, E.K.: Robust constraint on Lorentz violation using fermi-LAT gamma-ray burst data. Phys. Rev. D 99, 083009 (2019)

Publisher's note Springer Nature remains neutral with regard to jurisdictional claims in published maps and institutional affiliations.

\section{Affiliations}

L. Burderi $^{1}$ - A. Sanna ${ }^{1}$ (D) . T. Di Salvo ${ }^{2}$ - A. Riggio ${ }^{1} \cdot$ R. laria ${ }^{2}$ A. F. Gambino $^{2}$ A. Manca $^{1}$ - A. Anitra ${ }^{2}$. S. M. Mazzola ${ }^{1}$ - A. Marino ${ }^{1}$
A. Sanna
andrea.sanna@dsf.unica.it
1 Dipartimento di Fisica, Università degli Studi di Cagliari, SP Monserrato-Sestu km 0.7, I-09042 Monserrato, Italy
2 Dipartimento di Fisica e Chimica, Università degli Studi di Palermo, via Archirafi 36, I-90123 Palermo, Italy 